\title{
Evaluation of episcleral venous fluid wave during gonioscopy assisted transluminal trabeculotomy in patients with advanced glaucoma
}

\author{
Zeynep Aktas $\mathbb{1}^{1} \cdot$ M. Cuneyt Ozmen ${ }^{1} \cdot$ H. Tuba Atalay ${ }^{1} \cdot$ A. Yucel Ucgul ${ }^{1}$
}

Received: 13 January 2018 / Revised: 7 May 2018 / Accepted: 25 June 2018 / Published online: 10 December 2018

(c) The Royal College of Ophthalmologists 2018

\begin{abstract}
Purpose To evaluate episcleral venous fluid wave (EVFW) during GATT surgery in patients with advanced stage open angle glaucoma and to investigate its relationship with the severity of glaucoma.

Methods In all, 34 eyes of 28 open angle glaucoma patients who underwent GATT surgery were included into study. The extent of EVFW was evaluated using surgical records of patients and it was defined as number of clock hours. Correlations between the extent of EVFW and GAAT surgical outcomes as determined by intraocular pressures (IOP) and postoperative antiglaucoma medication needs were investigated. The impact of preoperative maximum IOP on EVFW was also evaluated. Results There was a significant correlation between IOPs and the extent of the EVFW at 1st, 3rd, 6th and 12th months follow-up visits. 10 of (\%29.4) 34 eyes required antiglaucoma medications after the surgery. Mean extents of EVFW in patients who needed and did not need medications during follow-ups were $2.6 \pm 1.0(1-4)$ and $5.9 \pm 1.5(3-8)$ clock hours and the difference was statistically significant. Cutoff value of $<4.5$ clock hours for the EVFW (sensitivity $79.2 \%$, specifity $100 \%$ ) was found to be associated with the need for additional antiglaucoma medication.

Conclusions The extent of EVFW might be a valuable prognostic indicator for the surgical success of GATT since trabeculotomy is circumferential in this surgical technique making all collector channels accessible.
\end{abstract}

\section{Introduction}

Whereas trabeculectomy remains the most commonly performed glaucoma surgery worldwide, microinvasive glaucoma surgery (MIGS) is rapidly gaining ground due to its safer surgical profile. Even though very low target intraocular pressures (IOP) may be necessary for advanced glaucoma and achievable with trabeculectomy, a considerable number of sight threatening complications may occur including hypotonia, retinal detachment and endophthalmitis [1]. This is one of the main reasons for performing MIGS, which has a safer profile. It is easily combined with cataract surgery and is most typically indicated for patients with mild to moderate glaucomatous disease [2].

Zeynep Aktas, MD, FEBO

drzeynep2000@yahoo.com

1 Gazi University, Faculty of Medicine, Department of Ophthalmology, Ankara, Turkey
Schlemm's canal surgeries have the advantage of less invasive approach. It offers an improved outflow pathway by either bypassing or removing diseased trabecular meshwork (TM). As described by Grover et al. [3-5], gonioscopy-assisted transluminal trabeculotomy (GATT) procedure is a MIGS technique in which TM is circumferentially bypassed via the suture or catheter. In this technique, aqueous humor passes through the collector canals and episcleral veins through the cleaved open diseased TM.

In filtrating surgery, the main indicator for the success of the surgery is subconjunctival bleb morphology. However, for MIGS techniques, there are no such markers since these techniques are blebless procedures.

Fellman et al. [6]. described brand new peroperative marker for the success of the Trabectome (Neomedix Corp, Tustin, CA) procedure which they named as "Episcleral Venous Fluid Wave (EVFW). They found significant negative correlation between postoperative IOP and the extent of the EVFW. In the current study, we aimed to investigate the extent of the EVFW in patients who underwent GATT surgery and its relationship with the severity of the glaucoma. 


\section{Methods}

Retrospective chart review was performed in open angle glaucoma patients who underwent GATT surgery. Institutional ethical committee approval was taken. All patients underwent gonioscopic examination to verify that there was an open angle with visible angle landmarks especially trabecular meshwork. All surgeries were performed by one surgeon (ZA) at Gazi University Department of Ophthalmology, Ankara, Turkey and were recorded. Written consent was obtained from all patients. EVFW was evaluated using surgical videos in a masked fashion. All open angle glaucoma patients who underwent GATT surgery with good surgical record qualities were included. Patients with records in which there was a difficulty to evaluate the EVFW because of the overlying blood or chemosis were excluded.

After a standart sterile preparation of the patient, eye was draped and lid speculum was placed. The head of the patient was elevated slightly to decrease episcleral venous pressure. Temporal and superior paracentesis was placed using $23-\mathrm{G}$ microsurgical blade. A viscoelastic material (sodium hyaluronate) was injected into anterior chamber (AC) through temporal site incision. Microscope and the patient's head then oriented to allow ideal visualization of the gonioscopic structures under the view of the Swan-Jacob goniolens. A 1-2 mm goniotomy was created using a microvitreoretinal (MVR) blade through the temporal site paracentesis. 5-0 prolene suture was blunted by the hand-held cautery and was introduced into $\mathrm{AC}$ through the superior quadrant paracenthesis toward the angle. MVR forceps was then introduced through the same temporal site and the blunted tip of the 5-0 prolene suture was grasped with MVR forceps and then introduced into the Schlemm's canal. MVR forceps was also used to advance the suture in the SC circumferentially. During this step, the progress of the suture was easily followed since the color of the suture is dark blue and it is easy to see it in the SC even behind the highly pigmented TM (Fig. 1).

In majority of cases, suture passed circumferentially and then the distal tip of was grasped with microsurgical forceps again. While holding the distal tip of the prolene, the proximal part of the suture was pulled until the time circumferential ab-interno trabeculotomy was completed. If suture stops somewhere in the SC, another paracentesis was created in the inferior quadrant to make it circumferential again in other direction.

At the end of the surgery, viscoelastic material was removed by bimanual irrigation-aspiration system and episcleral veins were checked for any BSS flow and/or blanching during maximum irrigation. This flow is called EVFW as described by Fellmann et al [6]. Only the extent of the EVFW in terms of clock hours was determined by

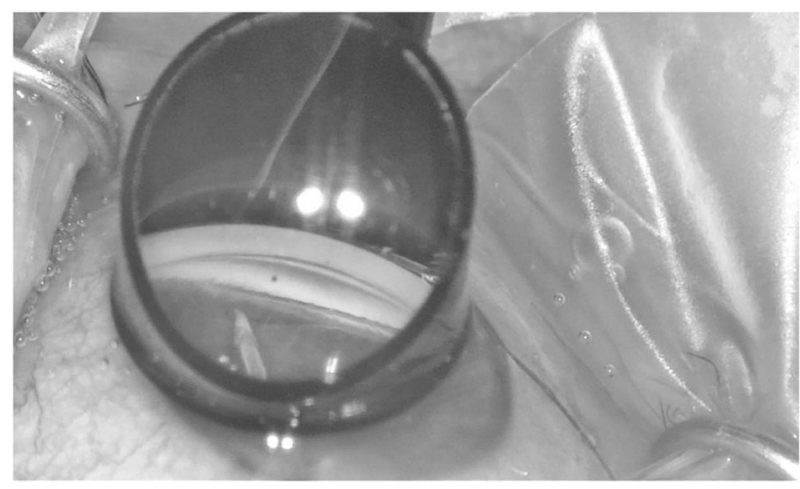

Fig. 1 5-0 prolene suture in the Schlemm's canal circumferentially

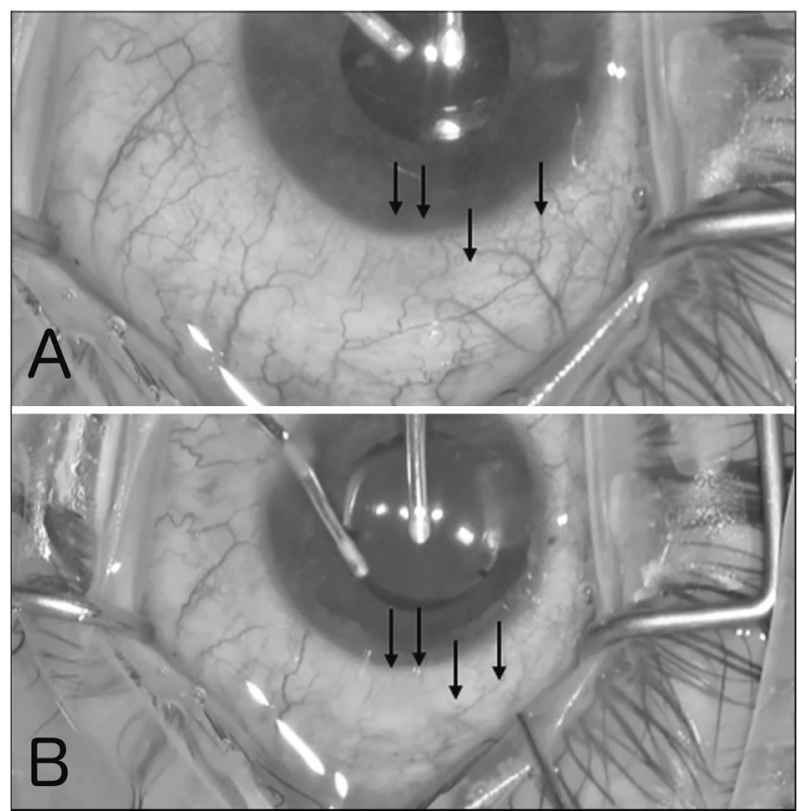

Fig. 2 Intraoperative appearance of episcleral veins when there is no flow in the anterior chamber, foot position zero (a); blanching of episcleral veins during the maximal irrigation of the anterior chamber (b)

reviewing the surgical recordings (Fig. 2a, b). However, blanching could not be graded since it was hard to compare the amount of blanching in most of the surgical videos.

Preoperative and postoperative 1, 3, 6, 12 months IOPs and the number of medications were recorded. Correlations between EVFW and mean retinal nerve fiber layer thicknesses (RNFLT), IOP and number of medications were investigated.

The data obtained from the study were recorded using Excel for Windows (version 2010, Microsoft, Redmond, WA) and statistical analyses were performed using the Statistical Package for the Social Sciences for Windows (version 21.0, SPSS, Chicago, IL). Pearson or Spearman correlation coefficients evaluated the degree of correlation between the values, where it is appropriate. The statistical level of significance was set to $\mathrm{p}<0.05$. Continuous 
variables were computed as mean $\pm \mathrm{SD}$ and median (minmax) where applicable. Differences between preoperative and postoperative IOP and medications were assessed using paired-samples T-test. Differences between groups were assessed using Independent samples T-test. Receiver operating characteristic (ROC) curve was used to determine the predictive value of EVFW for postoperative IOP lowering medication use and to calculate the sensitivity and specifity.

\section{Results}

34 eyes of 28 patients who underwent GATT surgery were included in the study. All of the patients were open angle glaucoma patients comprising primary open angle glaucoma $(\mathrm{POAG})(n=18)$; pseudoexfoliative glaucoma $(\mathrm{XFG})(N=$ 10). Mean cup-to-disc ratio of patients was $0.76 \pm 0.12$ (0.6-0.9). Mean MD of patient's was $-19.7 \pm 8.3(-5.20$ to 33.0). However, we did not get reliable visual fields from all of the patients since some of them had significantly depressed visual fields and MDs of them were out of range. Mean lenght of time patients have been on drops was $3.5 \pm$ 1.4 year (1-6 year). Correlation between mean duration of antiglaucoma treatment and IOP was only significant in 1st month whereas not significant in $3^{\text {rd }}, 6^{\text {th }}$ and 12 th months, respectively ( $p: 0.033, r: 0.420 ; p: 0,949, r: 0.013 ; p: 0.148$, $r: 0.292 ; p: 0.323, r: 0.107)$. Furthermore, there was no significant correlation between mean duration of treatment and EVFW ( $p$ : $0.215 ; r$ : -0.251 ).

Mean baseline IOP and number of medications were found to be significantly decreased in all follow-ups (Table 1). However, postoperative antiglaucoma drops were still needed in 10 of 34 eyes (29.4\%).

There was statistically significant correlation between the number of clock hours of the EVFW and the postoperative IOP in 1 st month (p:0.003), $3^{\text {rd }}$ month ( $\left.p: 0.002\right)$, 6th month ( $p: 0.001)$ and 12th months $(p: 0.001)$. When the patients on antiglaucoma drops were excluded, same postoperative significant correlations were also detected on 1st, 3rd, 6th months but not on 12th months ( $p: 0.001, p: 0.035, p$ : $0.030, p$ : 0.184) (Fig. 3a-d).

Interestingly, the mean number of clock hours of the EVFW in patients with and without antiglaucoma medications were $2.6 \pm 1.0(1-4)$ and $5.9 \pm 1.5(3-8)$ clock hours respectively and the difference was statistically significant ( $p$ : 0.0001). Cutoff value of $<4.5$ clock hours of the EVFW with a sensitivity $79.2 \%$ and specifity $100 \%$ was found to be associated with the need for additional antiglaucoma medication ( $p<0.001$, AUC: 0.965 SE: 0.028) (Fig. 4).

There was a negative but statistically not significant correlation between the number of clock hours of the EVFW and the preoperative number of medications (p:0291, r:-0.186). On the other hand, there was no
Table 1 Baseline Demographics of the Patients

\begin{tabular}{ll}
\hline Patients, $n$ & 28 \\
Eyes, $n$ & 34 \\
Age, year & $52.5 \pm 12.6(20-68)$ \\
Sex, $n$ & \\
$\quad$ Famale & 13 \\
$\quad$ Male & 15 \\
Glaucoma type, $(n)$ & \\
$\quad$ Primary open angle glaucoma & 18 \\
$\quad$ Exfoliative glaucoma & 10 \\
Baseline IOP (mmHg) & $27.4 \pm 6.1(18-43)$ \\
Baseline number of antiglaucoma medications & $3.2 \pm 0.8(1-4)$ \\
Mean duration of antiglaucoma treatment (year, & $3.5 \pm 1.4(1-6)$ \\
n: 26) & \\
Follow-up (Months - number of eyes) & \\
$\quad$ Month 1 ( $n: 34)$ & \\
$\quad$ IOP & $14.4 \pm 4.5(7-30)$ \\
$\quad$ Number of antiglaucoma medications & $0.3 \pm 1.7(0-2)$ \\
Month 3 ( $n: 34)$ & $13.7 \pm 3.9(8-20)$ \\
$\quad$ IOP & $1.3 \pm 1.0(0-3)$ \\
$\quad$ Number of antiglaucoma medications & $0.5 \pm 0.5(0-2)$ \\
Month 6. $(n: 34)$ & \\
$\quad$ IOP & \\
$\quad$ Number of antiglaucoma medications & \\
$\quad$ Month 12. ( $n: 34)$ & $13.9 \pm 2.7(7-19)$ \\
$\quad$ IOP & \\
$\quad$ Number of antiglaucoma medications & \\
\hline
\end{tabular}

IOP: Intraocular pressure for the age, baseline and follow-up IOP / number of antiglaucoma medications, duration of antiglaucoma treatment data: mean $+/$ - SD (range)

significant correlation between the extent of EVFW and the mean RNFLT or MD values.

\section{Discussion}

In recent years, indications and the types of glaucoma surgeries have been changed. The major aim of these advancements is to reduce the complication rates, especially blebrelated issues. Schlemm's canal surgeries have the advantage of using patient's natural outflow pathways. I-stent, Trabectome, Hydrus implant surgeries are the main canal-based surgeries performed over the past decade [2, 7-10]. With these SC surgeries, treatment strategy for adult open angle glaucoma have been changed. Circumferential trabeculotomy surgeries have been started to be performed more compared to incisional conjunctival glaucoma surgery techniques in various glaucoma centers of the world. Aktas et al [11]. found the outcomes of external suture trabeculotomy comparable to trabeculectomy in adult open angle glaucoma in terms of IOP decrease and topical medication need. 

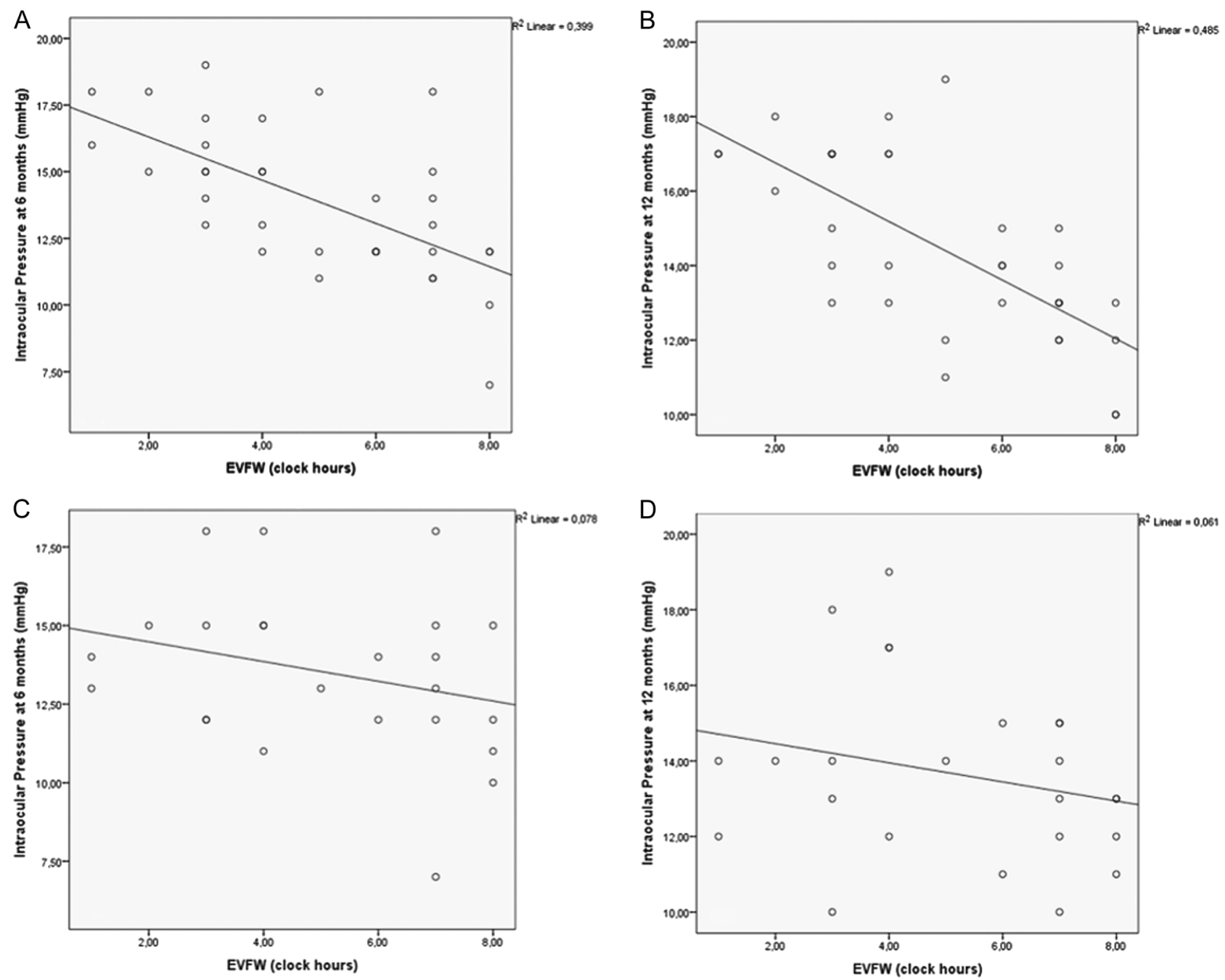

Fig. 3 Correlations between EVFW and postoperative IOP in all patients at 6 months (a) and 12 months (b) follow-ups; and in patients without antiglaucoma medications at 6 months (c) and 12 months (d) follow-ups

Grover et al. $[3,4]$ described GATT surgery as a suitable surgical technique for adult open angle and also juvenile glaucoma. Same team also described perioperative EVFW, for predicting the surgical success of the Trabectome surgery [6]. Trabectome surgery is also one of the canal-based surgeries that can be performed only in the nasal angle. Thus, they graded EVFW between 0-6 clock hours. They found significant correlation between the postoperative IOP - additional IOP lowering medication need and the degree of EVFW in terms of clock hours. In the current study, we investigated the relationship between same parameters in patients underwent GATT surgery which we believe this might have given more objective data since all collector canals were reached by this circumferential technique. We additionally investigate the relationship between glaucoma severity and EVFW which is not reported in the literature before.
Our data supports the data of Fellmann et al [6]. We found significant correlation between postoperative IOP and the extent of the EVFW in 3rd, 6th and 12th month followups. To prevent bias, we also checked for the same correlation once the patients on postoperative antiglaucoma medications were excluded just to see the real IOP-lowering effect of surgery and its relationship with EVFW. Thus, we found same correlation in except $12^{\text {th }}$ months follow-up. Fellmann et al [6]. reported the lack of a statistical correlation seen at 6 and 12 months follow-ups. They thought that this lack of significance might have been resulted from the subacute and chronic wound healing response in SC together with closure of the collector canals. In our study, lack of correlation on 12th months might be the result of the same reason.

We still do not know what causes SC to collapse and which factors reduce the success of SC surgery. However, 


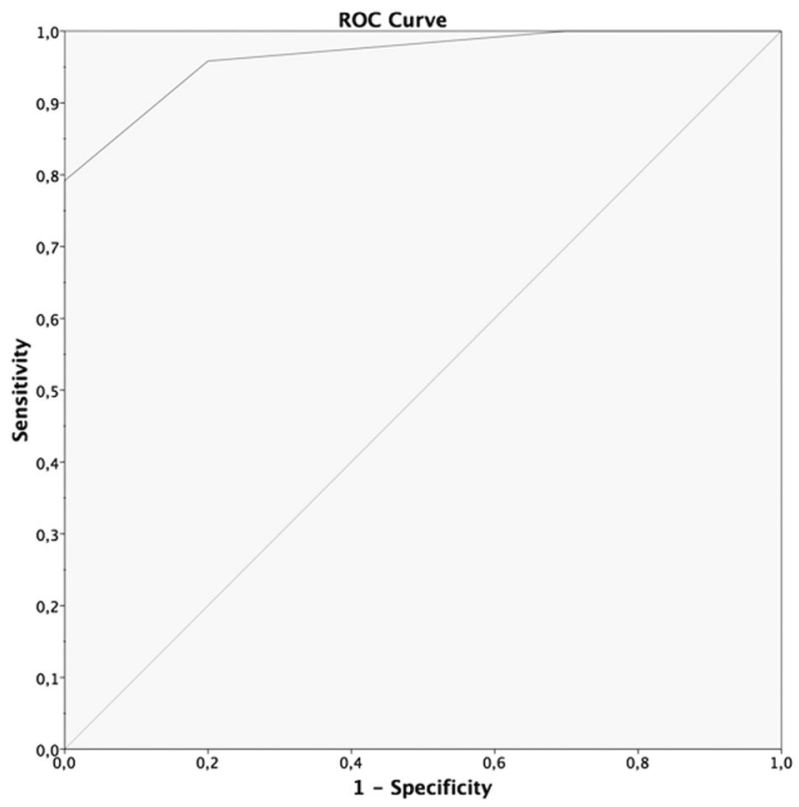

Fig. 4 Best EVFW cutoff value to predict postoperative antiglaucoma drop use was 4.5 (clock hours); receiver operating characteristic (ROC) curve

the stage of glaucoma and the number of medications might also affect the surgical success in terms of collector canal patency as shown by EVFW. Thus, we also investigated the relation between mean RNFLT, number of preoperative medications and the extent of EVFW. Mean number of medications might indirectly reflect the severity of glaucoma and might also effect the patency of the distal outflow system causing inflammation as they cause inflammation on the ocular surface. Unfortunately, we did not find any significant correlation between the RNFLT and the EVFW. This might be the result of our study population, which mostly included patients with advanced glaucoma. On the other hand, we also did not find any significant correlation between the MD and the EVFW. In our study, we included moderate to severe open angle glaucoma patients since our patient population does not tend to apply for medical care in earlier stages of the disease. This led us to see what would be the outcomes of GATT surgery in these patients. The mean $\mathrm{C} / \mathrm{D}$ ratio of our study population was $0.76 \pm 0.12$ (0.6-0.9) and the mean MD was $-19.7 \pm 8.3(-5.20$ to 33.0). On the other hand, MD was not reliable in all patients since some of the patients were in very advanced stage.

In our study population, as a new finding, additional medications after surgery was needed in $29.4 \%$ of the eyes. Furthermore, the number of clock hours of the EVFW was significantly lower in these patients compared to that of patients who were not on any medications after the surgery. According to ROC curve analysis patients with observed EVFW of more than 4.5 clock hours cutoff value did not seem to need any topical medication after the GATT surgery. This finding has not been reported in the literature, previously.

According the current literature, GATT seems to be more successful in mild to moderate glaucoma patients. Jordan JF et al. [12] reported that fewer patients with less severe glaucomatous damage required a further glaucoma surgery after Trabectome surgery. On the other hand Ahuja et al [13]. reported the outcomes of their Trabectome surgery in which they included patients with advanced glaucomatous damage with 0.9 cup-to-disc ratios. $28 \%$ of the advanced glaucoma patients needed further surgery whereas $10 \%$ of mild to moderate ones. In the current study, our patients were mostly composed of advanced glaucoma cases; 10 eyes $(29.4 \%)$ of all eyes needed IOP lowering medications after the surgery and 53\% of them had an advanced glaucoma with a MDs of higher than -12 . However total number of patients requiring further surgery was $2(5.4 \%$ of total) even though most of the patients were in advanced stage of the disease.

It has not well known currently what definitely causes collector channel atrophy. The most probable reason should be the advanced glaucoma as reported in the literature previously by Hann et al [14]. Trabecular meshwork herniation of the inner wall might reported to be cause of the obstruction of the collector canal openings. However, IOP and the stage of the disease might not be the only factor causing these anatomical changes and dysfunction leading to failure of the angle surgery. Because, although our patients mostly had an advanced glaucoma, we did not see surgical failure in these cases that much as reported previously in the current literature. GATT outcomes might also be related to mean duration of antiglaucoma treatment which is expected to be a long time in advanced disease. In the current study, most of the advanced patients were operated shortly after the first ophthalmic examination. Mean duration of antiglaucoma treatment was 3.5 years ( $n$ : 26). Relatively short duration of the medical treatment might be the reason for better surgical outcomes of our advanced glaucoma patients compared to the outcomes previously reported. On the other hand, there was no significant correlation between the mean duration of antiglaucoma treatment and postoperative IOPs/EVFW. Furthermore, we were not able to find direct correlation between the EVFW and MD or RNFLT. So these points should be clarified in future prospective studies including higher number of patients.

Ideally, we should be able to evaluate outflow preoperatively to discriminate which patient is suitable for GATT surgery. Currently, there is no preoperative technique predicting the success of the canal-based surgery. Huang et al [15]. recently described aqueous angiography technique in live human subjects which is promising. In this study, eight subject with nuclear or posterior subcapsular cataracts 
were included. During cataract surgery, indocyanine green (ICG) was injected into the $\mathrm{AC}$ as a capsular stain while simultaneously serving as a tracer for aqueous angiographic imaging. After imaging, standart phacoemulsification was performed in these patients. Aqueous angiography was performed using an angiographer on a customized arm (Spectralis HRA + OCT Flex module; Heidelberg Engineering, Heidelberg, Germany). With this system; they reported segmental aqueous outflow. They also confirmed angiographic signal with anterior segment OCT images showing larger intrascleral lumens compatible with aqueous humor outflow compared to angiographically negative regions.

In summary, parallel to the data of Fellman et al. [6], we also found that EVFW was an intraoperative indicator for also GATT surgery in which all collector channels were exposed. Same correlation on 3rd and 6th months followups was also found in patients who were not on postoperative antiglaucoma drops. Patients with limited EVFW less than mean 4.5 clock hours seems to need topical antiglaucoma medications after the GATT surgery. Furthermore, based on our data, GATT surgery, as a MIGS, might also be applicable for patients with advanced glaucoma. Additionally, low-teen target IOPs can also reached with the aid of topical medications if it cannot be achieved with only GATT surgery. We did not find any direct relationship between glaucoma severity and EVFW in patients with advanced glaucoma. Thus, further studies are still needed to shed light on the exact mechanisms and factors playing role in the surgical failure/success after GATT surgery especially in patients with advanced glaucoma. Additional researches also needed to see the aqueous outflow pathway preoperatively and to improve the clinical use of these canal based surgical techniques including GATT.

\section{Summary}

\section{What was known before}

- Episcleral venous fluid wave was found to be correlated with postoperative IOPs in mild to moderate glaucoma patients who underwent Trabectome surgery.

\section{What this study adds}

- Episcleral venous fluid wave has also been found to be correlated postoperative IOPs in patients underwent GATT surgery. Almost all of the patients that underwent GATT surgery in our study had advanced glaucoma, so this study also shows the relationship between EVFW and the outcomes of GATT, one of the MIGS surgeries, in advanced glaucoma.

\section{Compliance with ethical standards}

Conflict of interest The authors declare that they have no conflict of interest.

\section{References}

1. Gedde SJ, Schiffman JC, Feuer WJ, Herndon LW, Brandt JD, Budenz DL, et al. Treatment outcomes in the tube versus trabeculectomy (TVT) study after five years of follow-up. Am J Ophthalmol. 2012;153:789-803.

2. Saheb H, Ahmed IIK. Micro-invasive glaucoma surgery: current perspectives and future directions. Curr Opin Ophthalmol. 2012;23:96-104

3. Grover DS, Godfrey DG, Smith O, Feuer WJ, Montes de Oca I, Fellman RL. Gonioscopy-assisted transluminal trabeculotomy, ab internotrabeculotomy: technique report and preliminary results. Ophthalmology. 2014;121:855-61.

4. Grover DS, Smith O, Fellman RL, Godfrey DG, Butler MR, Montes de Oca I, et al. Gonioscopy assisted transluminal trabeculotomy: an ab interno circumferential trabeculotomy for the treatment of primary congenital glaucoma and juvenile open angle glaucoma. Br J Ophthalmol. 2015;99:1092-6.

5. Grover DS, Fellman RL. Gonioscopy-assisted transluminal trabeculotomy (GATT): thermal suture modification with a dyestained rounded tip. J Glaucoma. 2016;25:501-4.

6. Fellman RL, Feuer WJ, Grover DS. Episcleral venous fluid wave correlates with trabectome outcomes. Ophthalmology. 2015;122:2385-91.

7. Samuelson TW, Katz LJ, Wells JM, Duh YJ, Giaporcaro JE, US iStent Study Group. Randomized evaluation of the trabecular micro-bypass stent with phacoemulsification in patients with glaucoma and cataract. Ophthalmology. 2011;118:459-67.

8. Bacharach J. One year results of randomized controlled trial of cataract surgery with second generation trabecular microbypass stents in mild to moderate open-angle glaucoma. Abstract presented at ASCRS 2011 (Chicago) and AGS 2011 (New York City).

9. Saheb H, Johnstone MA, Toris CB, et al. A novel Schlemm'scanalscaffold maintains patency of of collector channel ostia in human anterior segments. Abstract, submitted to AGS (New York City) and ASCRS (Chicago); 2012.

10. Francis BA, Winarko J. Combined Trabectome and cataract surgery versus combined trabeculectomy and cataract surgery in open angle glaucoma. ClinSurgOphthalmol. 2011;29:4-10.

11. Aktas Z, Hepsen IF, Dilekmen N, Onol M. Are the outcomes of circumferential trabeculotomy for adult open angle glaucoma comparable to those of trabeculectomy? Clin Exp Ophthalmol. 2016;44:858-9.

12. Jordan JF, Wecker T, van Otrendorp C, Anton A, Reinhard T, Boehringer $\mathrm{D}$, et al. Trabectome surgery for primary and secondary open angle glaucomas. Graefes Arch Clin Exp Ophtalmol. 2013;251:2753-60.

13. Ahuja Y, Ma KhinPyi S, Malihi M, Hodge DO, Sit AJ. Clinical results of ab internotrabeculotomy using trabectome for open angle glaucoma: the Mayo clinic series in Rochester, Minnesota. Am J Ophthalmol. 2013;156:927-35.

14. Hann CR, Vercnocke AJ, Bentley MD, Jorgensen SM, Fautsch MP. Anatomic changes in Schlemm's canal and collector chennels in normal and primary open angle glaucoma eyes using low and high perfusion pressures. Invest Ophthalmol Vis Sci. 2014;55:5834-41.

15. Huang AS, Camp A, Xu BY, Penteado RC, Weinreb RN. Aqueous angiography: aqueous humor outflowimaging in live human subjects. Ophthalmology. 2017;124:1249-51. 